\title{
On Adaptive-Width Channel Allocation in Non-Cooperative, Multi-Radio Wireless Networks
}

\author{
Fan $\mathrm{Wu}^{\dagger}$ Nikhil Singh ${ }^{\star}$ Nitin Vaidya ${ }^{\star}$ Guihai Chen ${ }^{\dagger}$ \\ $\dagger$ Department of Computer Science and Engineering, Shanghai Jiao Tong University, China \\ * ECE \& Coordinated Science Lab, University of Illinois at Urbana-Champaign, USA \\ fwu@cs.sjtu.edu.cn; \{nsingh1, nhv\}@illinois.edu; gchen@nju.edu.cn
}

\begin{abstract}
Due to the limitation of radio spectrum resource and fast growing of wireless applications, careful channel allocation is highly needed to mitigate the performance degradation of wireless networks because of interference among different users. While most of the existing works consider allocating fixedwidth channels, combining contiguous channels may provide an alternative way to better utilize the available channels. In this paper, we study the problem of adaptive-width channel allocation from a game-theoretic point of view, in which the nodes are rational and always pursue their own objectives. We first model the problem as a strategic game, and show the existence of Nash equilibrium (NE), when there is no exogenous factor to influence players' behavior. We further propose a charging scheme to influence the players' behavior, by which the system is guaranteed to converge to a Dominant Strategy Equilibrium (DSE), a solution concept that gives participants much stronger incentives. We show that, when the system converges to a DSE, it also achieves global optimality, in terms of system-wide throughput without starvation. Numerical results verify that with our charging scheme, the system-wide throughput obtained is higher as compared to the throughput obtained when system is in NE.
\end{abstract}

\section{INTRODUCTION}

With the fast development of software defined radios, the problem of channel allocation, which assigns radio interfaces to available channels, is gaining increasing importance in wireless networks. For example, the current IEEE 802.11 [12] standard specifies several orthogonal channels (e.g., 3 such channels in IEEE 802.11b/g and 12 in IEEE 802.11a). Due to the limitation on the number of available channels, careful channel allocation is needed to mitigate the performance degradation of wireless networks because of interference among different users.

A recent study [6] shows that the width of IEEE 802.11based communication channels can be changed adaptively in software, even by using commodity Wi-Fi hardware. For example, in communication between a pair of radio interfaces, two contiguous $20 \mathrm{MHz}$ channels can be combined into a 40 $\mathrm{MHz}$ channel to provide higher throughput. Although much progress has been made in solving channel allocation problem in the literature, this finding makes the channel allocation problem more challenging - how to efficiently allocate radios to channels with adaptive width?

This work was supported in part by US NSF grant 06-27074, US Army Research Office grant W911NF-05-1-0246, and China NSF grants 60825205 and 61073152. The opinions, findings, conclusions, and recommendations expressed in this paper are those of the authors and do not necessarily reflect the views of the funding agencies or the governments.
In this paper, we study the problem of adaptive-width channel allocation from a game-theoretic perspective, in which the nodes in the wireless network are rational and always pursue their own objectives. We first model the problem as a strategic game, and show the existence of Nash equilibrium (NE), which is a fundamental solution concept from game theory, when there is no exogenous factor to influence players' behavior. However, NE does not provide an ideal solution to the problem of adaptive-width channel allocation in many cases. There are two reasons: (1) NE is not a very strong solution concept. Specifically, when in a NE, a player of the game has incentives to keep its equilibrium strategy only under the assumption that all the other players are also keeping their equilibrium strategies. When this assumption is not valid, NE does not provide incentives for the game player. (2) More importantly, NE is usually not socially efficient, which means that the system performance is not optimized. Therefore, when the system converges to one of the NEs, it could be the case that some of the selfish players benefit at the cost of system performance degradation.

To overcome the weaknesses of NEs, we further present a charging scheme to influence the players' behavior, by which the system is guaranteed to converge to a Dominant Strategy Equilibrium (DSE). DSE ensures each player always has incentive to use the equilibrium strategy, regardless of the other nodes' strategies. We show that, when the system converges to the DSE, global optimality is also achieved. Here, by global optimality, we mean that the system-wide throughput is maximized under the condition of no starvation. Therefore, the DSE-based outcome is better than the above NE-based outcome.

The contributions of this paper are as follows:

- To our knowledge, we are the first to study the problem of adaptive-width channel allocation from a game-theoretic perspective, and provide a strong solution.

- We model the problem of adaptive-width channel allocation as a strategic game. The game model is general enough to capture the properties of the adaptive-width channel, and both single-radio and multi-radio nodes.

- We also present a charging scheme, for using the channels, to influence the players' behavior. We prove that, by using our charging scheme, the system is guaranteed to converge to a DSE, in which global optimality is achieved. 
The rest of the paper is organized as follows. In Section II, we present our system model and game model for the adaptivewidth channel assignment problem, and recall solution concepts used in this paper from game theory. In Section III, we prove the existence of NE without exogenous influence. In Section IV, we present a charging scheme to achieve a DSE with global optimality. In Section V, we report the evaluation results. In Section VI, we briefly review the related works. Finally, we conclude the paper and point out potential future works in Section VII.

\section{Model And Solution CONCEPTS}

In this section, we introduce our system model and game model for the adaptive-width channel allocation problem, and recall some useful solution concepts from game theory.

\section{A. System Model}

We consider a static wireless network, where each node is equipped with one or multiple radio interfaces. We assume these radios can be tuned to operate on channels of arbitrary width. As in papers [8], [29], we assume that pairs of nodes need to communicate with each other over a single hop, and each node participates in only one of the communication sessions. We assume that the communication pairs' packets are backlogged, which means that every communication pair has infinite packets to send. We denote the set of communication pairs by $N=\{1,2, \ldots, n\}$, where each element $i$ has an identification number. In this paper, we assume the identification numbers are 1 through $n$.

To communicate, a pair of nodes need to tune one or multiple radios to their shared channel(s). We require that a transmission must be between two radios, where one acts as transmitter, and the other acts as receiver. So we only consider the case in which both nodes of a communication pair allocate the same number of radios to each of their shared channel(s). A pair of nodes can have parallel transmissions between them, if they both allocate more than one radios. Here, let each communication pair $i \in N$ have $r_{i}$ radio pairs (i.e., both of the nodes have $r_{i}$ radios). The radio pair distribution vector is denoted by $R=\left(r_{1}, r_{2}, \ldots, r_{n}\right)$.

We assume there is a set of contiguous, orthogonal (noninterfering), and homogenous channels (e.g., 3 such channels in IEEE $802.11 \mathrm{~b} / \mathrm{g}$ and 12 in IEEE $802.11 \mathrm{a}$ ), denoted by $K$. The available channels are also numbered from 1 to $|K|$. We further assume that $r_{i} \leq|K|, \forall i \in N$. As shown in paper [6], off-the-shelf wireless radio can be tuned to a wider channel combined by contiguous channels, and the bit-rate on the combined channel is proportional to its bandwidth. We consider MAC layer protocol used are CSMA/CA based protocols (e.g., IEEE 802.11 standards). Let $B$ denote the bandwidth of a channel. We assume that we get a combined channel with bandwidth $\lambda B$ by combining $\lambda$ contiguous channels. Let $T\left(W_{c}, N_{c}\right)$ denote the effective aggregate throughput on channel $c$, where $c$ can be a single channel or a set of contiguous channels, $W_{c}$ is the bandwidth of channel $c$, and $N_{c}$ is the number of radio pairs competing for $c$. Here, we assume that all the $N_{c}$ radio pairs are using the same center frequency and channel width. We leave the case in which radio pairs can compete for partially overlapping channels, to our future work. We assume that the effective aggregate throughput $T\left(W_{c}, N_{c}\right)$ is a convex non-increasing function of $N_{c}$ for $N_{c}>0$, when the width of channel $W_{c}$ is fixed (refer to [4]); and $T\left(W_{c}, N_{c}\right)$ is a concave non-decreasing function of $W_{c}$ for $W_{c}>0$, when the number of competing radio pairs $N_{c}$ is fixed (refer to [6]). If $c=\emptyset$ or $N_{c}=0$, we define $T\left(W_{c}, N_{c}\right)=0$. Figure 1 illustrates the effective aggregate throughput $T\left(W_{c}, N_{c}\right)$ as a function of channel bandwidth $W_{c}$ and the number of competing radio pairs $N_{c}$.

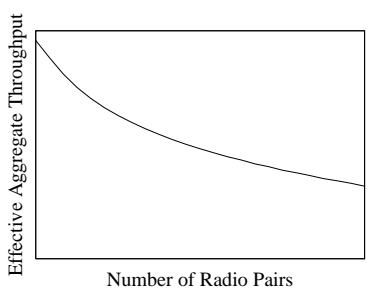

(a) Fixed channel bandwidth $W_{c}$

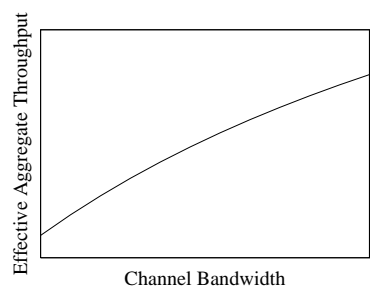

(b) Fixed number of competing radio pairs $N_{c}$.
Fig. 1. Effective aggregate throughput $T\left(W_{c}, N_{c}\right)$ as a function of channel bandwidth $W_{c}$ and the number of competing radio pairs $N_{c}$.

Since it is shown that the effective aggregate throughput on a channel $c$ can be shared equally among the radio transmitters using that channel [4], [5], we assume that each radio pair on channel $c$ gets a throughput $T\left(W_{c}, N_{c}\right) / N_{c}$, when $c \neq \emptyset$ and $N_{c}>0$.

In this paper, we only consider a single collision domain, wherein all transmissions on the same channel will collide with each other. Extending our work to multiple collision domains is left for future study. We assume there is a common control channel, on which the nodes can communicate with each other without involving other nodes as intermediate relays.

\section{B. Game Model}

We model the problem of adaptive-width channel allocation as a strategic game, namely channel allocation game.

In the channel allocation game, we consider the set $N$ of communication pairs as players. In the rest of the paper, we use player and communication pair interchangeably. We assume players do not collude/cooperate with each other. Each player $i \in N$ has $r_{i}$ radio pairs. The strategy of a player $i \in N$ is its radio-channel allocation vector:

$$
s_{i}=\left(s_{i, 1}, s_{i, 2}, \ldots, s_{i, c}, \ldots, s_{i,\left|K^{\prime}\right|}\right),
$$

where $K^{\prime}$ is a set of reorganized channels including single (original) channels and combined channels:

$$
\bigcup_{c \in K^{\prime}} c=K
$$

and $s_{i, c}$ is the number of radio pairs that player $i$ allocates on a channel $c \in K^{\prime}$. The reorganized channels in $K^{\prime}$ are also numbered from 1 to $\left|K^{\prime}\right|$. In this paper, we do not consider the case in which the nodes cooperatively decide how to reorganize the channels. This cooperation leads to a 
coalitional game. Instead, we assume that $K^{\prime}$ is computed, independent of players strategies, by the system administrator, and then broadcasted to the players via the control channel. We leave the problem of channel allocation in cooperative wireless network to our future work. In contrast to the process of channel reorganization, channel allocation is done in a distributed manner.

The strategy profile $s$ is a matrix composed of all the players' strategies:

$$
s=\left(s_{1}, s_{2}, \ldots, s_{n}\right)^{T} .
$$

As a notational convention, $s_{-i}$ represents the strategy profile of all players except player $i$. Note that $s=\left(s_{i}, s_{-i}\right)$ is a complete strategy profile, in which player $i$ takes strategy $s_{i}$ and the other players take strategy profile $s_{-i}$.

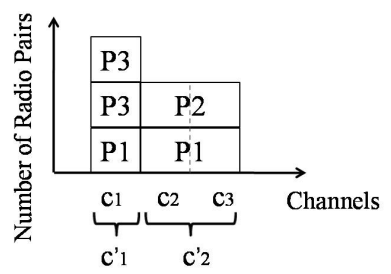

Fig. 2. A toy example of channel allocation, where $N=\left\{P_{1}, P_{2}, P_{3}\right\}$, $K^{\prime}=\left\{c_{1}^{\prime}, c_{2}^{\prime}\right\}$ (here, $c_{1}^{\prime}=\left\{c_{1}\right\}, c_{2}^{\prime}=\left\{c_{1}, c_{2}\right\}$ ), and $r_{1}=r_{2}=r_{3}=2$.

Figure 2 shows an example of channel allocation, with 3 players $N=\left\{P_{1}, P_{2}, P_{3}\right\}$ and 3 original channels $K=$ $\left\{c_{1}, c_{2}, c_{3}\right\}$. Each player has 2 radio pairs. Channel 2 and channel 3 are combined into a larger channel, resulting in a new channel set $K^{\prime}=\left\{c_{1}^{\prime}, c_{2}^{\prime}\right\}$, where $c_{1}^{\prime}=\left\{c_{1}\right\}$ and $c_{2}^{\prime}=\left\{c_{1}, c_{2}\right\}$. In Figure 2, player 1 places its 2 radio pairs on channel $c_{1}^{\prime}$ and the combined channel $c_{2}^{\prime}$, respectively; player 2 only use 1 radio pair and tunes it to the combined channel $c_{2}^{\prime}$; player 3 tune both of its radio pairs to $c_{1}^{\prime}$. Hence, the strategies taken by the players are: $s_{1}=(1,1), s_{2}=(0,1)$, and $s_{3}=(2,0)$.

Given a strategy profile $s$, the number of radio pairs used by a player $i$ cannot be larger than its "radio constraint":

$$
\sum_{c \in K^{\prime}} s_{i, c} \leq r_{i}, \forall i \in N .
$$

Here, the inequality indicates that it is not necessary for a player to use up all its radio pairs. Similarly, the number of radio pairs competing for a channel $c$ is:

$$
N_{c}(s)=\sum_{i \in N} s_{i, c}
$$

Hence, the throughput a player $i$ gets from a channel $c$ is:

$$
T_{i, c}(s)=\frac{s_{i, c}}{N_{c}(s)} \cdot T\left(W_{c}, N_{c}(s)\right),
$$

and the total throughput a player $i$ gets is:

$$
T_{i}(s)=\sum_{c \in K^{\prime}} T_{i, c}(s)
$$

Finally, the system throughput is:

$$
T(s)=\sum_{i \in N} T_{i}(s)=\sum_{c \in K^{\prime}} T\left(W_{c}, N_{c}(s)\right) .
$$

We assume that the players are rational and their objective is to maximize their own utilities. We denote the utility of a player $i \in N$ by $u_{i}$ :

$$
u_{i}(s)=v_{i}(s)-p_{i}(s),
$$

where $v_{i}(s)$ is player $i$ 's valuation on the outcome of the strategy profile $s$, and $p_{i}(s)$ is a charge for using the channels. For simplicity, we assume the valuation is proportional to the player's throughput:

$$
v_{i}(s)=\alpha T_{i}(s),
$$

where $\alpha$ is a positive constant coefficient. A well-designed charging formula can influence players' strategies and achieve good system-wide performance. In Section III, we study the NE the system converges to when there is no charging scheme to influence players' strategies (i.e., $p_{i}(s)=0$ ). In Section IV, we present a charging scheme, and show that the system converges to a DSE, in which global optimality is also achieved.

\section{Solution Concepts}

In this section, we review some solution concepts used in this paper from game theory.

Definition 1 (Nash Equilibrium [21]): A strategy profile $s^{\star}$ is a Nash Equilibrium of a strategic game, if for any player $i \in N$ and any strategy $s_{i} \neq s_{i}^{\star}$,

$$
u_{i}\left(s_{i}^{\star}, s_{-i}^{\star}\right) \geq u_{i}\left(s_{i}, s_{-i}^{\star}\right) .
$$

Definition 2 (Dominant Strategy Equilibrium [9], [21]): A strategy profile $s^{\star}$ is a dominant strategy equilibrium of a strategic game, if for any player $i \in N$, any strategy $s_{i} \neq s_{i}^{\star}$, and any strategy profile of the other players $s_{-i}$,

$$
u_{i}\left(s_{i}^{\star}, s_{-i}\right) \geq u_{i}\left(s_{i}, s_{-i}\right) .
$$

In reality, any practical solution to the channel allocation game should satisfy some additional requirements. First of all, there should not be any starvation. Second, it should satisfies social efficiency, which means that the system-wide throughput should be maximized. Integrating these two requirements, we define the concept of global optimality for a solution ${ }^{1}$.

Definition 3: (Global Optimality) In a channel allocation game, suppose that $s^{\star}$ is a strategy profile of channel allocation. We say $s^{\star}$ is globally optimal if the following two requirements are met:

1) No starvation: $T_{i}\left(s^{\star}\right)>0, \forall i \in N$.

2) Social efficiency: $T\left(s^{\star}\right) \geq T(s), \forall s \neq s^{\star}$, if $s$ satisfies requirement (1).

We note that the globally optimal channel allocation might not be unique. But all globally optimal channel allocations have the same system-wide throughput.

\footnotetext{
${ }^{1}$ Our definition of global optimality is slightly different from a traditional definition, which usually considers the optimization of a single metric (e.g., throughput).
} 


\section{EXISTENCE OF NASH EQUiLIBRIUM}

In this section, we study the existence of Nash equilibrium, when there is no charging scheme to influence players' strategies. Hence, each player's objective becomes solely maximizing its throughput:

$$
u_{i}(s)=\alpha T_{i}(s) .
$$

We present an algorithm to compute a NE channel allocation strategy profile. The Nash equilibrium approach in this section is obtained using some modifications to the Nash equilibrium scheme in [8].

Due to space limitations, we omit the proofs in this paper. Please refer to an extended version of the paper [27] for details of our proofs.

\section{A. Computing $N E$}

Since contiguous channels can be combined into a wider channel in our model, we require that the computed set of reorganized channels should contain all the original channels.

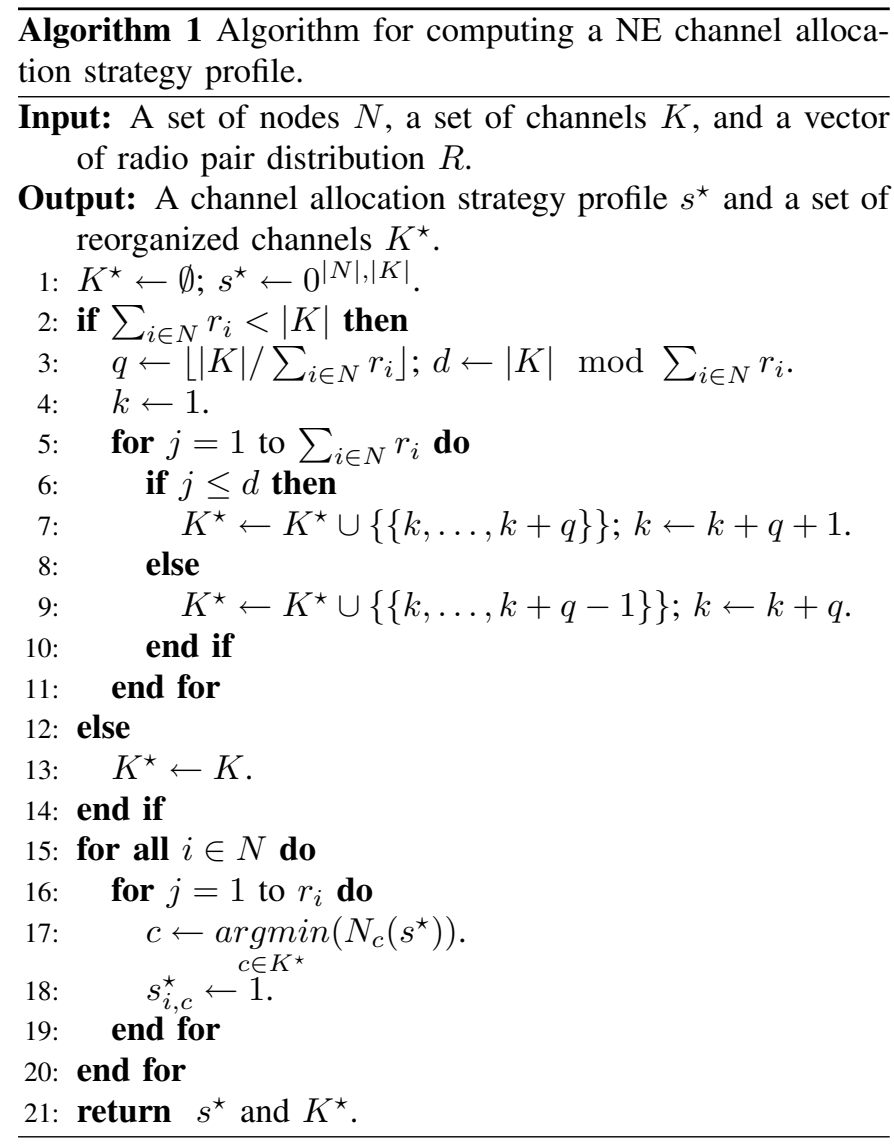

Algorithm 1 shows the pseudo-code of our algorithm for computing a NE channel allocation strategy profile. In lines $2-14$, we reorganize the channels by combining contiguous channels, if the total number of radio pairs is less than that of the channels, such that $\sum_{i \in N} r_{i} \geq\left|K^{\star}\right|$. In this case, we create $d$ combined channels, each containing $\left\lfloor|K| / \sum_{i \in N} r_{i}\right\rfloor+1$ original channels, and $\sum_{i \in N} r_{i}-d$ (combined) channels, each containing $\left\lfloor|K| / \sum_{i \in N} r_{i}\right\rfloor$ original channels. In lines 15-20, we evenly distribute all the radio pairs to the reorganized channels, and ensure that each player has at most 1 radio pair on any channel. Here, $\operatorname{argmin}()$ function is a deterministic function. When there is a tie, the function return the channel with the smallest identification number. Finally, the algorithm returns a NE channel allocation strategy profile $s^{\star}$ and a set of reorganized channels $K^{\star}$.

\section{B. Analysis}

In this section, we prove that the channel allocation strategy profile $s^{\star}$ computed by Algorithm 1 is a NE on the set of reorganized channels $K^{\star}$. We distinguish two cases in our analysis.

1) $\sum_{i \in N} r_{i}<|K|$ : The total number of radio pairs is less than the number of channels.

2) $\sum_{i \in N} r_{i} \geq|K|$ : The total number of radio pairs is no less than the number of channels.

We first consider the case, where the total number of radio pairs is less than the number of channels, i.e., $\sum_{i \in N} r_{i}<$ $|K|$. Considering the properties of the effective aggregate throughput function $T\left(W_{c}, N_{c}\right)$, to maximize the system-wide throughput, we should combine the original channels into $\sum_{i \in N} r_{i}$ channels and allocate one radio pair to each of the reorganized channels. Due to the concavity of the effective aggregate throughput function $T\left(W_{c}, N_{c}\right)$ when $N_{c}$ is fixed, the maximal system-wide throughput is achieved only when the original channels are evenly distributed into the combined channels:

$$
\max \left\{W_{c} \mid c \in K^{\star}\right\}-\min \left\{W_{c} \mid c \in K^{\star}\right\} \leq B .
$$

We also show that it is a NE by allocating one radio pair to each of the reorganized channels in $K^{\star}$.

Lemma 1: When $\sum_{i \in N} r_{i}<|K|$, channel allocation $s^{\star}$ on channel set $K^{\star}$ is a Nash equilibrium, if $N_{c}\left(s^{\star}\right)=1, \forall c \in K^{\star}$.

When $\sum_{i \in N} r_{i}<|K|$, we have $\sum_{i \in N} r_{i}=\left|K^{\star}\right|$, and Algorithm 1 allocates exactly one radio pairs to each channel in $K^{\star}$. Therefore, $s^{\star}$ and $K^{\star}$ computed by Algorithm 1 satisfy Lemma 1, when $\sum_{i \in N} r_{i}<|K|$.

We now consider the other case, where the total number of radio pairs is no less than that of the channels, i.e., $\sum_{i \in N} r_{i} \geq$ $|K|$. In this case, we do not combine any channel $\left(K^{\star}=K\right)$. A sufficient condition for NE was shown in [8]:

Lemma 2: [8] When $\sum_{i \in N} r_{i} \geq|K|$, a channel allocation $s^{\star}$ on channel set $K^{\star}$ is a Nash equilibrium, if it satisfies the following two conditions:

1) $\left|N_{c}-N_{c^{\prime}}\right| \leq 1, \forall c, c^{\prime} \in K^{\star}$.

2) $s_{i, c}^{\star} \leq 1, \forall i \in N, \forall c \in K^{\star}$.

Noting that line 17 and line 18 of Algorithm 1 indicate the two conditions in Lemma 2, respectively, $s^{\star}$ and $K^{\star}$ computed by Algorithm 1 also satisfy the sufficient condition shown in Lemma 2, when $\sum_{i \in N} r_{i} \geq|K|$.

Combining Lemma 1 and Lemma 2, we can get the following conclusion.

Theorem 1: The channel allocation strategy profile $s^{\star}$ computed by Algorithm 1 is a NE on channel set $K^{\star}$. 


\section{Achieving Global Optimality}

As we have mentioned, NE has its weaknesses, and global optimality may not be achieved in NEs. Figure 3 shows an example, in which global optimality can be achieved by using another channel allocation, instead of the NE channel allocation computed in the previous section.

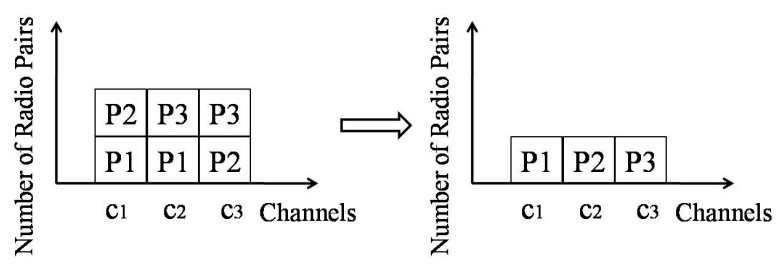

Fig. 3. An example of channel allocations, showing that system-wide throughput achieved by NE (left) can be improved by minimizing the number of players on each channel, using a better channel allocation (right). Here $N=\left\{P_{1}, P_{2}, P_{3}\right\}, K=\left\{c_{1}, c_{2}, c_{3}\right\}$, and $r_{1}=r_{2}=r_{3}=2$.

In this section, we overcome NE's weaknesses by introducing a carefully designed charging scheme, by which the system is guaranteed to achieve DSE. Hence, each player's objective is maximizing its utility, which is the difference between the player's valuation on its throughput and the charge for using the channels:

$$
u_{i}(s)=\alpha T_{i}(s)-p_{i}(s) .
$$

We also show that the DSE achieves the global optimality in terms of system-wide throughput, under the condition of no starvation.

In [29], the authors presented a charging scheme, which also ensures the system converges to DSE with global optimality ${ }^{2}$. However, their solution does not consider adaptive-bandwidth channels. Thus some of the channels are left unused if the number of radio pairs is less than that of the channels. Furthermore, when the other players follow the DSE channel allocation strategy profile, a player's utility is always nonpositive, which may hurt the player's incentives to participate the channel allocation game. In contrast, we propose a charging scheme to guarantee that a player can always get positive utility by following the DSE strategy, no matter what strategies the other players take.

\section{A. Charging Scheme}

Our objective is to make the system converge to a globally optimal channel allocation. However, if we allow the players to choose the channels arbitrarily, most likely the system would either not converge at all, or converge to an state that is not globally optimal, as shown in the previous section. Therefore, we need to introduce a scheme in order to influence the strategies of the players. Here the scheme we use is to charge the players for using the channels.

As in the literature (e.g., [7], [25], [30]-[32]), we assume that there is a virtual currency in the system. Each player has to pay some virtual money to the system administrator based

\footnotetext{
${ }^{2}$ In [29], the authors proved that the achieved equilibrium is a Strongly Dominant Strategy Equilibrium, which is a subset of DSE.
}

on the outcome of the channel allocation game. We regard this charge as the fee for using the channels.

We now assume that we have a globally optimal channel allocation $s^{\star}$ on channel set $K^{\star} .{ }^{3}$ (We will explain how to compute $s^{\star}$ in Section IV-B.)

In reality, a charging scheme for the channel allocation game should satisfy some additional requirements.

1) The charge to each player should be non-negative. In other words, the system administrator should not pay out any virtual money for the channel allocation.

$$
\forall i \in N, p_{i} \geq 0 .
$$

2) Given the globally optimal channel allocation $s^{\star}$, the total charges should not be larger than the value of the globally optimal system-wide throughput.

$$
\sum_{i \in N} p_{i} \leq \alpha T\left(s^{\star}\right)
$$

Before introducing the charging scheme, we need to define a strategy subtraction operation " $\ominus$ " between two strategies of a player $i$ on channel set $K^{\star}$ :

$$
s_{i} \ominus s_{i}^{\prime} \stackrel{\text { def }}{=}\left(\left(s_{i, c}-s_{i, c}^{\prime}>0\right) ?\left(s_{i, c}-s_{i, c}^{\prime}\right): 0\right)_{c \in K^{\star}} .
$$

Here, the strategy subtraction operation calculates the difference between each pair of corresponding elements in the two strategies. If the difference is not positive, then the operation lets the result element be 0 . Intuitively, the strategy subtraction operation calculates the difference between two channel allocation strategies, without resulting in any negative number of radio pairs allocated onto a channel.

Let $\hat{s}_{i}$ denote the difference between player $i$ 's strategy $s_{i}$ and its strategy in the globally optimal channel allocation $s^{\star}$ :

$$
\hat{s}_{i}=s_{i} \ominus s_{i}^{\star} .
$$

We now define the charge of player $i$ as follows:

$$
p_{i}(s)=\alpha\left(T\left(s_{i} \ominus \hat{s}_{i}, s_{-i}\right)-T(s)+\bar{T}_{i}(s)+\hat{T}_{i}(s)\right),
$$

where

$$
\begin{gathered}
\bar{T}_{i}(s)=\sum_{c \in K^{\star} \wedge N_{c}(s)=\hat{s}_{i, c}} \hat{s}_{i, c} T\left(W_{c}, 1\right), \\
\hat{T}_{i}(s)=\sum_{c \in K^{\star}}\left(\frac{\hat{s}_{i, c}}{N_{c}(s)} \cdot T\left(W_{c}, N_{c}(s)\right)\right) .
\end{gathered}
$$

Intuitively, there are three parts within bigger parentheses in the charging formula. The first part $T\left(s_{i} \ominus \hat{s}_{i}, s_{-i}\right)-T(s)$ calculates the degradation of system-wide throughput, if the player $i$ deviate from $s_{i}^{\star}$ by allocating extra radio pairs specified in $\hat{s}_{i}$ to the channels. We note that this part can

\footnotetext{
${ }^{3}$ Given a globally optimal channel allocation, a straightforward way to enforce the channel allocation is to punish any deviating player with an infinite charge. However, such an approach may hurt the players' willingness to participate the channel allocation game. For example, a player may not deliberately deviate, but may happen to somewhat deviate from the globally optimal channel allocation, because her information is incomplete or inconsistent with others when computing the channel allocation. Therefore, we present an alternative charging scheme in this paper.
} 
be negative if the player $i$ increases system-wide throughput by using strategy $s_{i}$ instead of $s_{i}^{\star}$, when the other players take strategy profile $s_{-i}$. This happens when the player $i$ luckily allocates an extra radio pair to a free channel (i.e., a channel not occupied by any other player). The second part $\bar{T}_{i}(s)$ represents the throughput got by the player $i$ using extra radio pairs specified in $\hat{s}_{i}$ on the free channel(s). Puting the first two parts together, we get the throughput degradation on channels that are not free. Since the second part cancels the possible system-wide throughput increment achieved by player $i$ by allocating radios to the free channels, we note that the sum of first two parts is always non-negative. The third part $\hat{T}_{i}(s)$ is the total throughput got by the extra radio pairs specified in $\hat{s}_{i}$. We also note that, if $s_{i}=s_{i}^{\star}$, then $p_{i}=0$; otherwise, $p_{i} \geq 0$.

Before showing that the channel allocation strategy profile $s^{\star}$ is a DSE on channel set $K^{\star}$, we first show the following lemma.

Lemma 3: If a channel allocation strategy profile $s^{\star}$ is globally optimal on channel set $K^{\star}$, then $s_{i, c}^{\star} \in\{0,1\}, \forall i \in$ $N, \forall c \in K^{\star}$.

Based on Lemma 3, we get the following theorem.

Theorem 2: If the above charging scheme is used, then it is a DSE when each player $i$ takes channel allocation strategy $s_{i}^{\star}$ on channel set $K^{\star}$.

By Theorem 2, it is straightforward to conclude that, if $s^{\star}$ is a globally optimal channel allocation strategy profile, then the DSE achieved is also globally optimal.

\section{B. Globally Optimal Channel Allocation}

To implement the DSE, every player must share the same algorithm for computing the globally optimal channel allocation strategy profile $s^{\star}$ and the set of reorganized channels $K^{\star}$. In this section, we present an algorithm that requires perfect information of the network. We assume every player can obtain such information by broadcasting beacons, containing one's identification number and number of radio pairs, and listening beacons from the other players, in the control channel.

Algorithm 2 shows the pseudo-code of our algorithm for computing a globally optimal channel allocation strategy profile. The first part of the algorithm (lines 2-14) is identical to that of Algorithm 1, which reorganize the channels according to the total number of radio pairs, such that $\sum_{i \in N} r_{i} \geq\left|K^{\star}\right|$. Next, the algorithm evenly distribute the players onto the reorganized channels (lines 15-18). If the number of players is less than that of the channels, the algorithm keeps allocating each unoccupied channel with a player who still has unused radio pair, until all the channels are occupied (lines 19-26). For fairness, the algorithm tries to make every player has almost the same number of radio pairs allocated, unless it does not has sufficient number of radio pairs. Finally, the algorithm returns a globally optimal channel allocation strategy profile $s^{\star}$ and a set of reorganized channels $K^{\star}$.

It is straightforward to see the correctness of Algorithm 2, because the algorithm always fully utilizes all the channels and causes minimal system-wide throughput degradation, while ensuring that no player get starved.

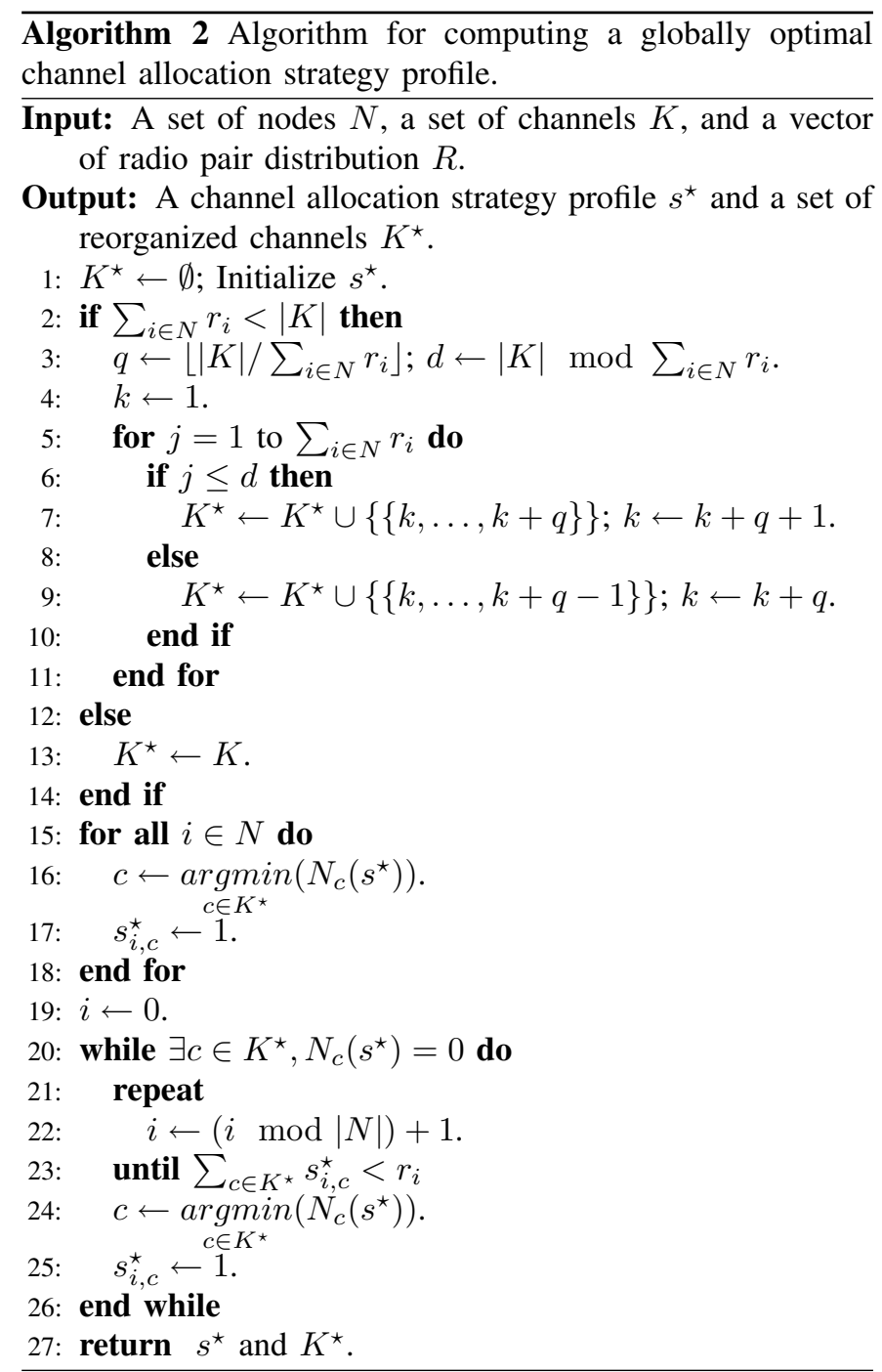

Theorem 3: The channel allocation strategy profile $s^{\star}$ and the set of reorganized channels $K^{\star}$ computed by Algorithm 2 is globally optimal.

\section{Numerical Results}

We evaluate the channel allocation outcome achieved in the NE and the DSE. The objective of our simulations is twofold. One is to verify that our charging scheme indeed overcomes the weaknesses of NE, and ensures that the system achieves DSE. The other one is to measure the influence of our scheme on the system performance, in terms of system-wide throughput.

\section{A. Methodology}

In the evaluations, a basic CSMA/CA protocol with binary slotted exponential back-off is used for MAC layer protocol. The values of the parameters used to obtain numerical results are listed in Table I. Our charging scheme is applied only when evaluating the DSE, while not for evaluating the NE.

Node Behavior: In our evaluations, we compare two kinds of node behavior: 
TABLE I

PARAMETERS USED TO OBTAIN NUMERICAL RESULTS

\begin{tabular}{|l|l|}
\hline Packet Payload & 1450 bytes \\
PHY\&MAC Header & 50 bytes \\
ACK Packet Size & 30 bytes \\
\hline Minimum Contention Window & 32 \\
Number of Backoff Stages & 5 \\
\hline Original Channel Bit Rate & $1 \mathrm{Mbps}$ \\
Propagation Delay & $1 \mu \mathrm{s}$ \\
Slot Time & $50 \mu \mathrm{s}$ \\
SIFS & $28 \mu \mathrm{s}$ \\
DIFS & $128 \mu \mathrm{s}$ \\
ACK Timeout & $300 \mu \mathrm{s}$ \\
\hline$\alpha$ & 1 \\
\hline
\end{tabular}

- Following NE/DSE: Following one's corresponding strategy in the NE/DSE channel allocation strategy profile computed by our algorithms.

- Deviating NE/DSE: Unilaterally deviating from one's corresponding strategy in the NE/DSE channel allocation strategy profile computed by our algorithms. For each of the radio pairs, the deviating player randomly chooses to use or not use the channel with equal probabilities.

Metrics: We evaluate two metrics:

- Utility: Utility is the difference between the player's valuation on throughput and charge for using the channels.

- System-wide throughput: System-wide throughput is the sum of all the players' throughputs.

\section{B. Impact of Player's Behavior on Its Utility}

In our first set of simulations, we evaluate the impacts of a player's behavior on its own with or without our charging scheme, and compare the strength of the incentives provided by NE and DSE. In this set of simulations, we assume there are 20 players and 12 channels in the system. The number of radio pairs on each player is uniformly selected in $[1,3]$. Each simulation is repeated 1000 times. Due to the limitation of space, we only show the results of the first 50 runs. In each run, we record the players' utilities obtained by following NE/DSE and deviating from NE/DSE, while the other players' strategy profile does not change.

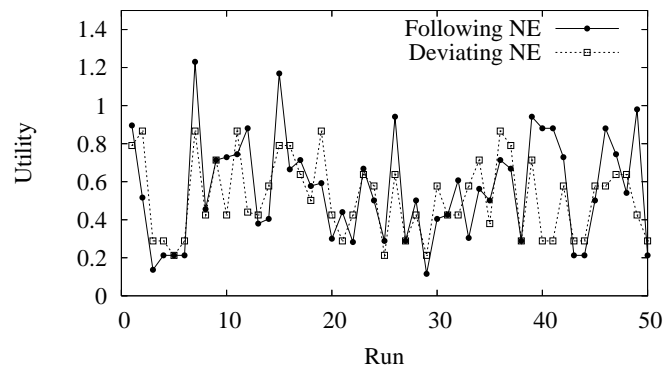

Fig. 4. Utilities of player 6 obtained by following the NE vs. deviating from the NE, when the other players deviate from the NE channel allocation strategy profile.

Figure 4 shows the utilities of a randomly selected player (player 6) obtained by following the NE and deviating from the NE, when the other players deviate from the NE channel allocation strategy profile. We note that the results for the other players are similar to that of player 6 . The figure shows that, in some runs, the utility of following the NE is no less than that of deviating from the NE; while in the other runs, deviating is better than following. This result shows that NE cannot provide any incentive for a player, when the other players do not follow the NE strategy profile.

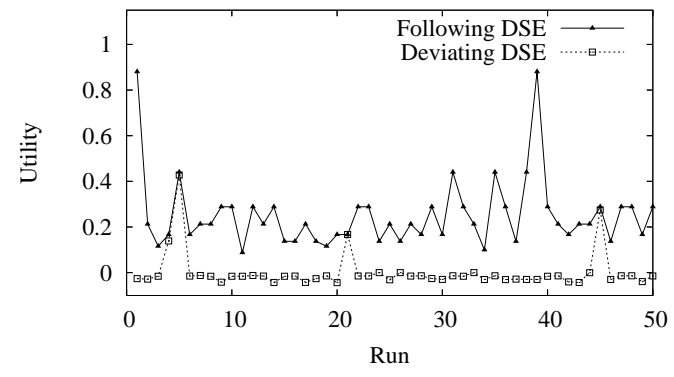

Fig. 5. Utilities of player 6 obtained by following the DSE vs. deviating from the DSE, when the other players deviate from the DSE channel allocation strategy profile.

Figure 5 shows the utilities of a the same player (player 6) obtained by following the DSE and deviating from the DSE, when the other players deviate from the DSE channel allocation strategy profile. The figure shows that the utility of a player while following the DSE is always no lower than that of deviating. Furthermore, the utility of following is always positive, while that of deviating is negative most of the time. Therefore, when our charging scheme is used, the incentives for using the DSE strategy is always guaranteed, no matter what the other players' strategy profile is. This result verifies that players cannot benefit by deviating from the DSE channel allocation strategy profile, when our charging scheme is used. So that our charging scheme indeed guarantee the convergence to the DSE.

We observe that the peak utility of a player in Figure 4 is higher than that in Figure 5 (i.e., 1.23 in Figure 4 and 0.88 in Figure 5). This is because a player always wants to use up all of its radio pairs, when our charging scheme is not applied and there exist other competing player. However, this higher peak utility is achieved as a cost of system-wide throughput degradation, which will be shown in the next set of evaluations.

\section{Performance of the Channel Allocation Game}

Our second set of simulations is to evaluate system performance, in terms of system-wide throughput, when our charging scheme is applied or not. As shown in previous sections, with our charging scheme, the system converges to a DSE; otherwise, the system converges to a NE. Here, we use DSE-based and NE-based outcomes to represent the results of the two cases, respectively. In the evaluation, we repeat each simulation until the convergence level $10^{-6}$ is reached.

Figure 6 shows the system-wide throughputs achieved by NE-based and DSE-based outcomes as a function of number of players, where the number of channels is 12 and the number of radio pairs on each player is randomly selected 


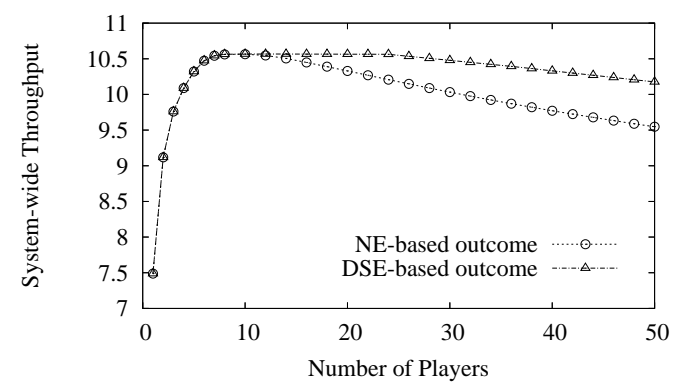

Fig. 6. System-wide throughputs achieved by NE-based and DSE-based outcomes as a function of number of players, when the number of channels is 12 and the average number of radio pairs per player is 2 .

in $[1,3]$. When the number of players is no more than 12 (resource is relatively abundant to competitors), NE-based and DSE-based outcomes achieve almost the same systemwide throughput. However, the selfish nature of the players makes the performance of NE-based outcome more and more worse than that of DSE-based outcome, with the growth of the number of players. Specifically, when there are 50 players, the system-wide throughput of the DSE-based outcome is 0.63 Mbps (or 6.59\%) higher than that of the NE-based outcome.

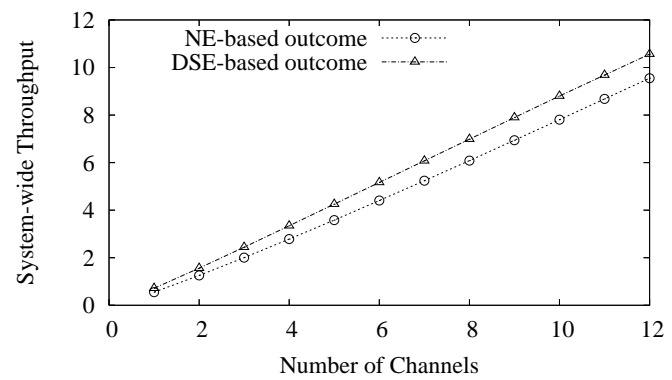

Fig. 7. System-wide throughputs achieved by NE-based and DSE-based outcomes as a function of number of channels, when the number of players is 20 and the average number of radio pairs per player is 5 .

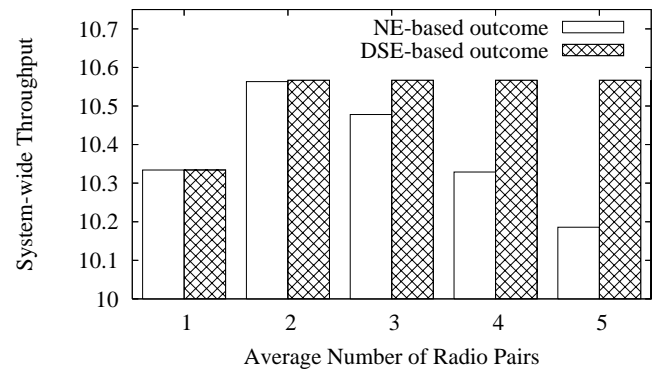

Fig. 8. System-wide throughputs achieved by NE-based and DSE-based outcomes for different average numbers of radio pairs per player, when the number of players and channels are is 10 and 12, respectively.

Figure 7 and Figure 8 show the system-wide throughputs achieved by NE-based and DSE-based outcomes as a function of number of channels and average number of radio pairs per player, respectively, when the other factors are fixed. Again, these results show that DSE-based outcome always performs at least as good as that of NE-based outcome.

The above results show that DSE-based outcome is more desirable than NE-based outcome. In other words, the usage of our charging scheme is important in improving system-wide throughput.

\section{RELATED WORK}

In this section, we first review related works on channel allocation that assume cooperation of participants, and then review the works with selfish participants.

\section{A. Cooperative Channel Allocation}

The channel allocation problem was first studied in cellular networks. We refer to [13] for a comprehensive survey.

A number of works were presented for wireless LANs (WLANs). For instance, Mishra et al. [18] utilized weighted graph coloring to address channel allocation for WLANs. Mishra et al. [19] used client-driven mechanisms to address the joint problem of channel allocation and load balancing in centrally managed WLANs.

Channel allocation problems are also studied in wireless mesh networks (WMNs). For example, Alicherry et al. [1], Raniwala et al. [23], and Kodialam et al. [14] considered channel allocation together with routing or scheduling in order to maximize network throughput. Some other works (e.g., [22]) focused on the channel allocation problem in rural mesh networks built with directional antennas.

The channel allocation problem is also studied in other wireless networks, such as ad-hoc networks (e.g., [16]) and software defined radio networks (e.g., [11]).

\section{B. Channel Allocation with Selfish Participants}

The related works described in VI-A require that all nodes in the network must be cooperative. Here cooperative means that the nodes unconditionally obey a central control or behave strictly according to prescribed protocol. However, this assumption is not valid when the network consists of selfish nodes, whose goals are to maximize their payoff. With the existence of selfish nodes, assigning radios to channels becomes a game.

In an earlier work, Felegyhazi et al. [8] studied Nash Equilibria in a static multi-radio multi-channel allocation game. Later, $\mathrm{Wu}$ et al. [29] proposed a mechanism to make the multi-radio multi-channel allocation game converge to a much stronger equilibrium state, called strongly dominant strategy equilibrium (SDSE), in which optimal system throughput is achieved. Both of these works considered the problem in a single collision domain. Recently, a number of strategyproof auction-based spectrum allocation mechanisms (e.g., TRUST [34], VERITAS [33], and SMALL [28]) have been proposed to solve the channel allocation problem in multiple collision domain. Another important related work on channel allocation game is [10], in which the authors proposed a graph coloring game model and discussed the price of anarchy under various topology conditions such as different channel numbers and bargaining strategies. However, none the the above work considers adaptive-width channels. 
In wireless networks, game theoretic approaches are also used to study media access problems. For example, MacKenzie et al. [17] studied the selfish behavior of nodes in Aloha networks. Later, Cagalj et al. [5] and Konorski [15] used gametheoretic approaches to investigate the media access problem of selfish nodes in CSMA/CA networks. In cognitive radio networks, Nie and Comaniciu [20] proposed a game theoretic framework to analyze the behavior of cognitive radios for distributed adaptive spectrum allocation.

There are also other works on incentive-compatibility in wireless networks. Examples include those works on packet routing and forwarding in ad hoc networks [2], [3], [7], [24][26], [30]-[32].

\section{CONCLUSion AND Future Work}

In this paper, we have modeled the problem of adaptivewidth channel allocation as a strategic game, and have studied the existence of NE, when no external factor is given to influence the players' strategies. Furthermore, we have presented a strategy-influencing charging scheme to ensure the system converge to a DSE, in which global optimality is reached. Evaluation results show that our charging scheme achieves good performance as expected.

As for future work, there are several potential directions. One direction is to extend our current work to a multihop scenario, while achieving good economic properties and system performance. Another direction is to study the channel allocation game, when nodes can compete for partially overlapping channels.

\section{REFERENCES}

[1] M. Alicherry, R. Bhatia, and L. Li, "Joint channel assignment and routing for throughput optimization in multi-radio wireless mesh networks," in MobiCom'05, Sep. 2005.

[2] L. Anderegg and S. Eidenbenz, "Ad hoc-VCG: a truthful and costefficient routing protocol for mobile ad hoc networks with selfish agents," in MobiCom'03, Sep. 2003.

[3] N. Ben Salem, L. Buttyan, J. P. Hubaux, and M. Jakobsson, "A charging and rewarding scheme for packet forwarding in multi-hop cellular networks,' in MobiHoc'03, Jun. 2003.

[4] G. Bianchi, "Performance analysis of the IEEE 802.11 distributed coordination function," IEEE Journal on Selected Areas in Communications, vol. 18, no. 3, pp. 535-547, 2000.

[5] M. Čagalj, S. Ganeriwal, I. Aad, and J.-P. Hubaux, "On selfish behavior in CSMA/CA networks," in INFOCOM'05, Apr. 2005.

[6] R. Chandra, R. Mahajan, T. Moscibroda, R. Raghavendra, and P. Bahl, "A case for adapting channel width in wireless networks," in SIGCOMM'08, Seattle, WA, Aug. 2008.

[7] S. Eidenbenz, G. Resta, and P. Santi, "Commit: A sender-centric truthful and energy-efficient routing protocol for ad hoc networks with selfish nodes,' in IPDPS'05, Apr. 2005.

[8] M. Félegyházi, M. Čagalj, S. S. Bidokhti, and J.-P. Hubaux, "Noncooperative multi-radio channel allocation in wireless networks," in INFOCOM'07, May 2007.

[9] D. Fudenberg and J. Tirole, Game Theory. MIT Press, 1991.

[10] M. M. Halldórsson, J. Y. Halpern, L. E. Li, and V. S. Mirrokni, "On spectrum sharing games," in PODC'04, Jul. 2004
[11] Y. T. Hou, Y. Shi, and H. D. Sherali, "Optimal spectrum sharing for multi-hop software defined radio networks," in INFOCOM'07, May 2007.

[12] IEEE 802.11 Working Group, "IEEE Std 802.11-2007 IEEE Standard Part 11: Wireless LAN Medium Access Control (MAC) and Physical Layer (PHY) Specifications," 2007.

[13] I. Katzela and M. Naghshineh, "Channel assignment schemes for cellular mobile telecommunications: A comprehensive survey," IEEE Personal Communications, vol. 3(3), pp. 10-31, Jun. 1996.

[14] M. Kodialam and T. Nandagopal, "Characterizing the capacity region in multi-radio multi-channel wireless mesh networks," in MobiCom'05, Sep. 2005.

[15] J. Konorski, "Multiple access in ad-hoc wireless lans with noncooperative stations," in IFIP Networking'02, May 2002.

[16] P. Kyasanur and N. Vaidya, "A routing protocol for utilizing multiple channels in multi-hop wireless networks with a single transceiver," in QShine'05, Aug. 2005.

[17] A. B. MacKenzie and S. B. Wicker, "Stability of multipacket slotted Aloha with selfish users and perfect information," in INFOCOM'03, Apr. 2003.

[18] A. Mishra, S. Banerjee, and W. Arbaugh, "Weighted coloring based channel assignment for WLANs," ACM SIGMOBILE Mobile Computing and Communications Review (MC2R), vol. 9, no. 3, pp. 19-31, 2005.

[19] A. Mishra, V. Brik, S. Banerjee, A. Srinivasan, and W. Arbaugh, "A client-driven approach for channel management in wireless LAN," in INFOCOM'06, Apr. 2006.

[20] N. Nie and C. Comaniciu, "Adaptive channel allocation spectrum etiquette for cognitive radio networks," in DySPAN'05, Nov. 2005.

[21] M. J. Osborne and A. Rubenstein, A Course in Game Theory. MIT Press, 1994.

[22] B. Raman, "Channel allocation in 802.11-based mesh networks," in INFOCOM'06, Apr. 2006.

[23] A. Raniwala, K. Gopalan, and T. cker Chiueh, "Centralized channel assignment and routing algorithms for multi-channel wireless mesh networks," ACM SIGMOBILE Mobile Computing and Communications Review (MC2R), vol. 8, no. 2, pp. 50-65, 2004.

[24] V. Srinivasan, P. Nuggehalli, C.-F. Chiasserini, and R. Rao, "Cooperation in wireless ad hoc networks," in INFOCOM'03, Apr. 2003.

[25] W. Wang, S. Eidenbez, Y. Wang, and X.-Y. Li, "Ours-optimal unicast routing systems in non-cooperative wireless networks,' in MobiCom'06, Sep. 2006.

[26] W. Wang, X.-Y. Li, and Y. Wang, "Truthful multicast in selfish wireless networks," in MobiCom'04, Sep. 2004.

[27] F. Wu, N. Singh, N. Vaidya, and G. Chen, "On adaptive-width channel allocation in non-cooperative, multi-radio wireless networks," Technical Report, Nov. 2010.

[28] F. Wu and N. Vaidya, "Small: A strategy-proof mechanism for radio spectrum allocation," University of Illinois at Urbana-Champaign, Tech. Rep., 2010.

[29] F. Wu, S. Zhong, and C. Qiao, "Globally optimal channel assignment for non-cooperative wireless networks," in INFOCOM'08, Apr. 2008.

[30] S. Zhong, L. E. Li, Y. G. Liu, and Y. R. Yang, "On designing incentive-compatible routing and forwarding protocols in wireless adhoc networks-an integrated approach using game theoretical and cryptographic techniques," in MobiCom'05, Sep. 2005.

[31] S. Zhong, J. Chen, and Y. R. Yang, "Sprite, a simple, cheat-proof, creditbased system for mobile ad-hoc networks," in INFOCOM'03, Apr. 2003.

[32] S. Zhong and F. Wu, "On designing collusion-resistant routing schemes for non-cooperative wireless ad hoc networks," in MobiCom'07, Sep. 2007.

[33] X. Zhou, S. Gandhi, S. Suri, and H. Zheng, "ebay in the sky: Strategyproof wireless spectrum auctions," in MobiCom'08, Sep. 2008.

[34] X. Zhou and H. Zheng, "Trust: A general framework for truthful double spectrum auctions," in INFOCOM'09, Apr. 2009. 